PROCEEDINGS OF THE

AMERICAN MATHEMATICAL SOCIETY

Volume 128, Number 7, Pages 2165-2170

S 0002-9939(99)05186-2

Article electronically published on November 29, 1999

\title{
THERE ARE MANY KUNEN COMPACT L-SPACES
}

\author{
HENNO BRANDSMA AND JAN VAN MILL
}

(Communicated by Alan Dow)

\begin{abstract}
We prove that under $\mathrm{CH}$ there are $\omega_{1}$ non-homeomorphic Kunen compact L-spaces. Moreover there exist models of ZFC that have $2^{\omega_{1}}$ many non-homeomorphic Kunen spaces.
\end{abstract}

\section{INTRODUCTION}

In [5, Kunen constructed a compact L-space from CH. (For a similar construction under a somewhat weaker axiom, see [6].) His construction leaves a lot of freedom and the question naturally arises whether different constructions can yield topologically distinct spaces.

A different type of compact L-space is a Suslin continuum. Bell [1] proved that the hyperspace $H(X)$ of a monolithic Suslin continuum $X$ is monolithic. He asked whether for a compact monolithic hereditarily Lindelöf (HL) space $X$ it follows that $H(X)$ is monolithic. This question was answered by Brandsma and van Mill [2] in the negative by a modification of the Kunen construction from [5. This result was subsequently improved by the authors of that paper in 3]: every space defined like Kunen's example of a compact L-space has a non-monolithic hyperspace. It was left open whether there are non-homeomorphic Kunen compact L-spaces. The aim of this note is to prove that this is the case.

\section{KUnEN'S CONSTRUCTION}

In this section we briefly review the elements of Kunen's construction from [5] that are necessary for us.

Kunen's compact L-space is the limit $X_{\omega_{1}}$ of an inverse system $\left(X_{\alpha}, \pi_{\beta}^{\alpha}, \omega \leq \beta \leq\right.$ $\left.\alpha<\omega_{1}\right)$, such that for $\omega \leq \beta \leq \alpha, X_{\alpha}$ is a closed subspace of $2^{\alpha}$ and $\pi_{\beta}^{\alpha}: X_{\alpha} \rightarrow X_{\beta}$ is the restriction to $X_{\alpha}$ of the natural projection $2^{\alpha} \rightarrow 2^{\beta}$. In order to force the inverse limit $X_{\omega_{1}}$ of the system to be an L-space, some special conditions have to be met.

The first one is that for $\omega \leq \alpha<\omega_{1}, X_{\alpha}$ carries a Radon probability measure $\mu_{\alpha}$ such that

(K1) If $\beta \leq \alpha$, then $\mu_{\beta}=\mu_{\alpha}\left(\pi_{\beta}^{\alpha}\right)^{-1}$.

(K2) If $p \in X_{\alpha}$, then $\mu_{\alpha}(\{p\})=0$.

(K3) If $K \subseteq X_{\alpha}$ is non-empty and clopen, then $\mu_{\alpha}(K)>0$.

Received by the editors March 20, 1998 and, in revised form, August 5, 1998.

1991 Mathematics Subject Classification. Primary 54D35.

Key words and phrases. CH, L-space, measure. 
If $\omega \leq \gamma<\omega_{1}$ is a limit ordinal, then $X_{\gamma}$ is the inverse limit of the previous $X_{\alpha}$ 's, and by (K1) the measure $\mu_{\gamma}$ is determined by the previous measures $\mu_{\alpha}$ in the obvious way.

$X_{\omega}$ is $2^{\omega}$ with the usual product measure $\mu_{\omega}$.

If $\alpha=\beta+1$, then there is a closed subset $S_{\beta} \subseteq X_{\beta}$ such that

$$
X_{\alpha}=\left(X_{\beta} \times\{0\}\right) \cup\left(S_{\beta} \times\{1\}\right) .
$$

(Here, we are making the obvious identification of $2^{\alpha}$ with $2^{\beta} \times 2$.) The measure $\mu_{\alpha}$ is obtained from $\mu_{\beta}$ by splitting $S_{\beta}$ in half; thus, if $A \subseteq S_{\beta}$ is Borel, then

$$
\mu_{\alpha}(A \times\{0\})=\mu_{\alpha}(A \times\{1\})=\frac{1}{2} \mu_{\beta}(B)
$$

and if $A \subseteq X_{\beta} \backslash S_{\beta}$ is Borel, then $\mu_{\alpha}(A)=\mu_{\beta}(A)$.

The set $S_{\beta}$ is called the splitter at stage $\beta$.

Observe that $S_{\beta}$ is not arbitrary since (K3) forces it to be of positive measure everywhere. But this is not enough. If one puts $S_{\beta}=X_{\beta}$ for every $\omega \leq \beta<\omega_{1}$, then the inverse limit is homeomorphic to $2^{\omega_{1}}$, which is not an L-space. So the set $S_{\beta}$ must meet several other conditions. But an inspection of the construction in [5] yields that once $S_{\beta}$ has been identified meeting the desired demands, any nonempty closed set $T \subseteq S_{\beta}$ which is of positive measure everywhere will do equally well. This is the freedom in the construction that we mentioned in the introduction. So we will not concentrate on how to pick $S_{\beta}$ in order to get an L-space at the end: for this we refer to the construction [5]. But we will concentrate instead on the question of how a given splitter can be modified so as to get non-homeomorphic spaces.

\section{SHCHEPIN'S THEOREM}

Supppose that we have two inverse systems $\left(X_{\alpha}, \pi_{\beta}^{\alpha}, \omega \leq \beta \leq \alpha<\omega_{1}\right)$ and $\left(Y_{\alpha}, \rho_{\beta}^{\alpha}, \omega \leq \beta \leq \alpha<\omega_{1}\right)$ such as in the previous section.

By Shchepin's Theorem [7] it follows that each homeomorphism $f: X_{\omega_{1}} \rightarrow Y_{\omega_{1}}$ is induced in the following sense. There exists a closed and unbounded subset $C \subseteq\left[\omega, \omega_{1}\right)$ and for each $\alpha \in C$ a homeomorphism $f_{\alpha}: X_{\alpha} \rightarrow Y_{\alpha}$ such that the diagram

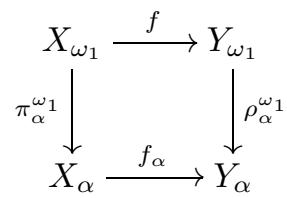

commutes. But this implies that if $\beta<\alpha$ are in $C$, then the diagram

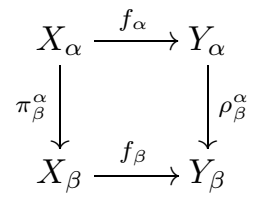

commutes.

The problem of constructing non-homeomorphic Kunen compact L-spaces boils down to the problem of how to destroy all possible commutative diagrams of the form (1). 


\section{The COnstruction}

In this section we will show how to get two non-homeomorphic Kunen spaces under $\mathrm{CH}$. In the next section we will show how this technique can yield more non-homeomorphic Kunen spaces. Applying CH, fix an enumeration $\left\{f_{\xi}^{\alpha}: \xi<\omega_{1}\right\}$ of all homeomorphisms between closed subsets of $2^{\alpha}$ when $\omega \leq \alpha<\omega_{1}$. Also, fix a function $g$ from $\omega_{1}$ onto $\omega_{1} \times \omega_{1}$ such that $g(\beta)=\langle\alpha, \xi\rangle$ implies $\alpha \leq \beta$.

Suppose that we are creating two Kunen L-systems $\left(X_{\alpha}, \pi_{\beta}^{\alpha}, \mu_{\alpha}, \omega \leq \beta \leq \alpha<\right.$ $\left.\omega_{1}\right)$ and $\left(Y_{\alpha}, \rho_{\beta}^{\alpha}, \hat{\mu}_{\alpha}, \omega \leq \beta \leq \alpha<\omega_{1}\right)$.

At stage $\beta$ of the construction, we perform the following test. Let $g(\beta)=\langle\alpha, \xi\rangle$. If $f_{\xi}^{\alpha}\left[X_{\alpha}\right] \neq Y_{\alpha}$, then we proceed with the construction of $X_{\beta+1}$ and $Y_{\beta+1}$ such as in [5]. There is work to be done if $f_{\xi}^{\alpha}\left[X_{\alpha}\right]=Y_{\alpha}$. We want to construct $X_{\beta+1}$ and $Y_{\beta+1}$ in such a way that for no $\gamma \geq \beta+1$ the diagram

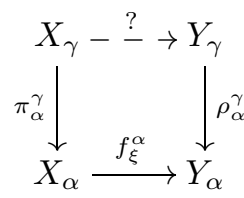

can be completed. This is done in the following way. Let $S \subseteq X_{\beta}$ and $T \subseteq Y_{\beta}$ be splitters for $X_{\beta}$ and $Y_{\beta}$, respectively, satisfying the demands in [5].

Put $S(\alpha)=\pi_{\alpha}^{\beta}[S]$. Observe that by (K1) and (K3) it follows that $\mu_{\alpha}(S(\alpha))>0$. As a consequence, $S(\alpha)$ is uncountable and hence contains a Cantor set, say $B_{X}$. Since each Cantor set contains a family consisting of $\mathfrak{c}$ pairwise disjoint Cantor sets, it is clear that we may assume that $\mu_{\alpha}\left(B_{X}\right)=0$. Consider the Cantor set $B_{Y}=f_{\xi}^{\alpha}\left[B_{X}\right]$. We may also assume that $\hat{\mu}_{\alpha}\left(B_{Y}\right)=0$. Consider the set $C_{Y}=$ $\left(\rho_{\alpha}^{\beta}\right)^{-1}\left[B_{Y}\right]$. This is a compact set with $\hat{\mu}_{\beta}$-measure 0 by (K1). Observe that its Cantor-Bendixson height is a countable ordinal number. (Recall that for a space the Cantor-Bendixson weight is the first ordinal number $\alpha$ such that the usual scattering process stops at stage $\alpha$ at a perfect set (possibly empty, but not in the (ase at hand).)

We now demand that our splitter $T_{\beta} \subseteq T$ misses the $\hat{\mu}_{\beta}$-nullset $C_{Y}$. It is clear that this is possible. In later steps $\gamma \geq \beta$ we demand that the splitter $T_{\gamma}$ misses the $\hat{\mu}_{\gamma}$-nullset $\left(\rho_{\beta}^{\gamma}\right)^{-1}\left[C_{Y}\right]$. This will cause no problems since at each stage we create only one "bad" nullset whose preimage has to be avoided by the respective splitter at each later step. This demand means that at each step of our construction the splitter at that step has to avoid an at most countable collection of nullsets coming from previous steps. So altogether this means that a nullset has to be avoided. But this can be done without any problem. Observe that this implies that for every $\gamma \geq \beta$ we have that $\left(\rho_{\alpha}^{\gamma}\right)^{-1}\left[B_{Y}\right]$ is homeomorphic to $C_{Y}$, and hence is of "small" Cantor-Bendixson height.

Put $C_{X}=\left(\pi_{\alpha}^{\beta}\right)^{-1}\left[B_{X}\right]$. The compact nullset $C_{X} \cap S$ is uncountable and hence contains a Cantor set, say $P$. Since $P$ is universal for compact zero-dimensional spaces, it contains a countable compact subspace $Z$ with larger Cantor-Bendixson height than the Cantor-Bendixson height of $C_{Y}$ (such sets are essentially countable ordinals with the order topology, see e.g. [8, II.8.6] for details). Observe that $C_{X} \cap S$, being a compact nullset, is a nowhere dense closed subset of $S$. It is therefore easy to construct a sequence $\left\{C_{n}: n<\omega\right\}$ consisting of pairwise disjoint non-empty 
clopen subsets of $S$ such that

1. $C_{n} \cap C_{X}=\emptyset$ for all $n$,

2. $\overline{\bigcup_{n<\omega} C_{n}}=\bigcup_{n<\omega} C_{n} \cup Z$.

Now put $S_{\beta}=\bigcup_{n<\omega} C_{n} \cup Z$ (note that the measure is positive everywhere on this set) and consider $X_{\beta+1}$. Observe that

$$
\left(S_{\beta} \times\{1\}\right) \cap\left(\pi_{\alpha}^{\beta+1}\right)^{-1}\left[B_{X}\right]=Z \times\{1\} .
$$

Since $S_{\beta} \times\{1\}$ is a clopen subset of $X_{\beta+1}$, this implies that the Cantor-Bendixson height of $\left(\pi_{\alpha}^{\beta+1}\right)^{-1}\left[B_{X}\right]$ is at least as large as the Cantor-Bendixson height of $Z$ which is larger than the Cantor-Bendixson height of $\left(\rho_{\alpha}^{\beta+1}\right)^{-1}\left[B_{Y}\right]$.

Since $\left(\pi_{\alpha}^{\beta+1}\right)^{-1}\left[B_{X}\right]$ is a nullset, we can and shall demand that its preimage will be avoided by the respective splitters at all later stages of the construction. That this can indeed be achieved follows by a similar reasoning as the one above. This means that for every $\gamma \geq \beta+1$ we obtain that $\left(\pi_{\alpha}^{\gamma}\right)^{-1}\left[B_{X}\right]$ is homeomorphic to $\left(\pi_{\alpha}^{\beta+1}\right)^{-1}\left[B_{X}\right]$, and hence is a set of "large" Cantor-Bendixson height.

Now if for some $\gamma \geq \beta+1, f: X_{\gamma} \rightarrow Y_{\gamma}$ is a homeomorphism making the diagram

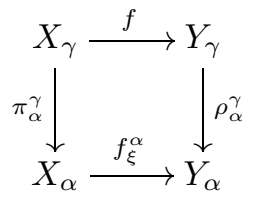

commute, then

$$
f\left[\left(\pi_{\alpha}^{\gamma}\right)^{-1}\left[B_{X}\right]\right]=\left(\rho_{\alpha}^{\gamma}\right)^{-1}\left[B_{Y}\right]
$$

which is impossible since by construction the corresponding Cantor-Bendixson heights do not match. This completes the construction.

Now assume that $f: X_{\omega_{1}} \rightarrow Y_{\omega_{1}}$ is a homeomorphism, and let $C \subseteq\left[\omega, \omega_{1}\right)$ be the closed and unbounded set promised by Shchepin's Theorem (see $\$ 3$ ). Let $\alpha$ be the minumum of $C$, and let $f_{\alpha}: X_{\alpha} \rightarrow Y_{\alpha}$ be the corresponding homeomorphism. There exists $\xi<\omega_{1}$ such that $f_{\alpha}=f_{\xi}^{\alpha}$. Let $\beta<\omega_{1}$ be such that $g(\beta)=\langle\alpha, \xi\rangle$. By construction, for no $\gamma \geq \beta+1$ the diagram

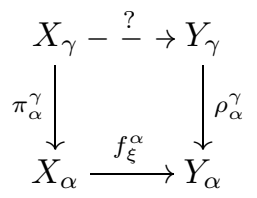

can be completed. Since $C$ is unbounded, Shchepin's Theorem implies that it can be completed beyond $\beta+1$. So we arrive at the desired contradiction.

\section{More nON-HOMEOMORPhiC Kunen SPACES}

Let us now sketch how to get $2^{\omega_{1}}$ many different Kunen spaces. In order to do this we assume, besides $\mathrm{CH}$, the existence of a Kurepa tree with $2^{\omega_{1}}$ many (cofinal) paths. These exist, e.g., under the axiom $\diamond^{+}$, which holds in the constructible universe (4, Cor. II. 7.11]). A Kurepa tree is a tree of height $\omega_{1}$, with all levels countable, but with at least $\omega_{2}$ many paths. These exist if and only if we have such a tree $T$ as a subtree of the binary tree of height $\omega_{1}$ (using Kurepa families as in [4]), so assume we have such a Kurepa subtree $T$. 
Let $\operatorname{Node}(T)$ denote the set of nodes of $T$, and for each $\beta<\omega_{1}$ let $T_{\beta}$ denote level $\beta$ of the tree. Fix a bijection $g: \omega_{1} \rightarrow \operatorname{Node}(T) \times \operatorname{Node}(T) \times \omega_{1}$ such that $g(\beta)=\langle s, t, \xi\rangle$ implies $s \neq t$ and $\operatorname{ht}(s)=\mathrm{ht}(t) \leq \beta$. Also construct by $\mathrm{CH}$ an enumeration $\left\{f_{\xi}^{s, t}: X_{s} \rightarrow X_{t} \mid \xi<\omega_{1}\right\}$ of all possible homeomorphisms between spaces that are at the same level in $T$. We do this inductively: the induction starts by taking all spaces at level $\omega$ to be $2^{\omega}$, and choosing the enumerations. Each time that we finished constructing another level of the tree we then choose enumerations for those spaces, and so on.

The plan is to build inverse limits that follow the paths of $T$, such that the limits over every path are non-homeomorphic. The basic idea will be the same as before. We will show that by the above technique we can "kill" countably many commutative diagrams at the same time: suppose that we are at level $\beta$ in our tree, and we want to construct level $\beta+1$ (as before there is no choice in the construction at limit levels). Let $g(\beta)=\langle s, t, \xi\rangle$, and let $f=f_{\xi}^{s, t}$. Define the sets $A$ and $B$ as follows: $A=\left\{u \in T_{\beta}: u\right.$ extends $\left.s\right\}$ and $B=\left\{u \in T_{\beta}: u\right.$ extends $\left.t\right\}$. We now have to destroy all possible arrows from spaces with index in $A$ to spaces with index in $B$. Because $T$ is a Kurepa tree, both sets are countable. If $u \in T_{\beta} \backslash(A \cup B)$, proceed with the construction of the next level (above $u$ ) as usual. For all pairs with $u \in A$ and $v \in B$ we can find null sets $C^{u, v}$ in $X_{v}$ that the splitter there must miss (these are the $C_{Y}$ from before). Now we let the splitter in $X_{v}$ miss the null set $\bigcup_{u \in A} C^{u, v}$. This can be done as before. For $X_{u}$ (with $u \in A$ ) we find, exactly as before, a splitter (inside the candidate splitter) that ensures that a certain inverse image has a Cantor-Bendixson height that exceeds the Cantor-Bendixson height of all the countably many sets $C^{u, v}$. In this way, if we keep on avoiding some null sets later on, we have killed all possible commutative diagrams with $f$. The proof of this is the same as before. This shows that all our $2^{\omega_{1}}$ many different paths all yield non-homeomorphic inverse limits.

Note that essentially the same proof will give $\omega_{1}=2^{\omega}$ many non-homeomorphic Kunen L-spaces under CH: just use a subtree of the binary tree of length $\omega_{1}$ that has countable levels, and $\omega_{1}$ many paths, e.g. take the tree that only has those sequences with finitely many ones.

Also note that $2^{\omega_{1}}$ is the maximal number of non-homeomorphic Kunen spaces. This a ZFC bound. This can be seen as follows: at every successor stage of the construction we choose some compact subset of a compact metrisable space to split. There are $2^{\omega}$ many choices possible for this, and the length of our inverse system is $\omega_{1}$. This yields $\left(2^{\omega}\right)^{\omega_{1}}=2^{\omega_{1}}$ many possible limits. The following question is a natural one:

Question. Does CH alone imply the existence of more than $\omega_{1}$ many non-homeomorphic Kunen spaces?

\section{REFERENCES}

[1] M. Bell, The Hyperspace of a Compact Space I, Topology and its Applications 72 (1996), 39-46. MR 97j:54010

[2] H. Brandsma and J. van Mill, A Compact HL Space need not have a Monolithic Hyperspace, Proceedings of the AMS 126 (1998), 3407-3411. MR 99a:54007.

[3] H. Brandsma and J. van Mill, Every Kunen-like L-space has a non-monolithic hyperspace, Proceedings of the 12th Summer Conference on General Topology and its Applications, 1997.

[4] K. Kunen, Set theory. An introduction to independence proofs, Studies in Logic and the foundations of Mathematics, vol. 102, North-Holland, Amsterdam, 1980. MR 82f:03001 
[5] K. Kunen, A Compact L-space under CH, Topology and its Applications 12 (1981), 283-287. MR 82h:54065

[6] K. Kunen and J. van Mill, Measures on Corson Compact Spaces, Fundamenta Mathematicae 147 (1995), 61-72. MR 96c:54040

[7] E. V. Ščepin, Functors and uncountable degrees of compacta, Uspekhi Mat. Nauk 36 (1981), no. 3(219), 3-62, 255. MR 82k:54012

[8] Z. Semadeni, Banach spaces of continuous functions, volume I, Monografie Matematyczne, vol. 55, PWN - Polish scientific publishers, Warsaw, 1971. 45:5730

Faculteit Wiskunde en Informatica, Vrije Universiteit, De Boelelaan 1081a, 1081 HV Amsterdam, the Netherlands

E-mail address: hsbrand@cs.vu.nl

E-mail address: vanmill@cs.vu.nl 